\title{
Association of Steroid 5a-Reductase Type 3 Congenital Disorder of Glycosylation With Early-Onset Retinal Dystrophy
}

\author{
Rachel L. Taylor, PhD; Gavin Arno, PhD; James A. Poulter, PhD; Kamron N. Khan, PhD, FRCOphth; \\ Jiten Morarji, BMBS, MSc; Sarah Hull, MA, FRCOphth; Nikolas Pontikos, PhD; Antonio Rueda Martin, PhD; \\ Katherine R. Smith, PhD; Manir Ali, PhD; Carmel Toomes, PhD; Martin McKibbin, FRCOphth; \\ Jill Clayton-Smith, MD, FRCP; Stephanie Grunewald, PhD, FRCPCH; Michel Michaelides, MD(Res), FRCOphth; \\ Anthony T. Moore, MA, FRCOphth; Alison J. Hardcastle, PhD; Chris F. Inglehearn, PhD; \\ Andrew R. Webster, MD(Res), FRCOphth; Graeme C. Black, DPhil, FRCOphth; \\ for the UK Inherited Retinal Disease Consortium and the 100,000 Genomes Project
}

IMPORTANCE Steroid 5a-reductase type 3 congenital disorder of glycosylation (SRD5A3-CDG) is a rare disorder of $\mathrm{N}$-linked glycosylation. Its retinal phenotype is not well described but could be important for disease recognition because it appears to be a consistent primary presenting feature.

OBJECTIVE To investigate a series of patients with the same mutation in the SRD5A3 gene and thereby characterize its retinal manifestations and other associated features.

DESIGN, SETTING AND PARTICIPANTS Seven affected individuals from 4 unrelated families with early-onset retinal dystrophy as a primary manifestation underwent comprehensive ophthalmic assessment, including retinal imaging and electrodiagnostic testing. Developmental and systemic findings were also recorded. Molecular genetic approaches, including targeted next-generation sequencing, autozygosity mapping, and apex microarray, were tried to reach a diagnosis; all participants were mutation negative. Whole-exome sequencing or whole-genome sequencing was used to identify the causative variant. Biochemical profiling was conducted to confirm a CDG type I defect. Patient phenotype data were collected over the course of ophthalmic follow-up, spanning a period of 20 years, beginning March 20, 1997, through September 15, 2016.

MAIN OUTCOMES AND MEASURES Detailed clinical phenotypes as well as genetic and biochemical results.

RESULTS The cohort consisted of 7 participants ( 5 females and 2 males) whose mean (SD) age at the most recent examination was 17.1 (3.9) years and who were all of South Asian ethnicity. Whole-exome sequencing and whole-genome sequencing identified the same homozygous SRD5A3 c.57G>A, p.(Trp19Ter) variant as the underlying cause of early-onset retinal dystrophy in each family. Detailed ocular phenotyping identified early-onset (aged $\leq 3$ years) visual loss (mean [SD] best-corrected visual acuity, +0.95 [0.34] logMAR [20/180 Snellen]), childhood-onset nyctalopia, myopia (mean [SD] refractive error, -6.71 [-4.22]), and nystagmus. Six of the 7 patients had learning difficulties and psychomotor delay. Fundus autofluorescence imaging and optical coherence tomographic scans were abnormal in all patients, and electrodiagnostic testing revealed rod and cone dysfunction in the 5 patients tested.

CONCLUSIONS AND RELEVANCE Mutations in the SRD5A3 gene may cause early-onset retinal dystrophy, a previously underdescribed feature of the SRD5A3-CDG disorder that is progressive and may lead to serious visual impairment. SRD5A3 and other glycosylation disorder genes should be considered as a cause of retinal dystrophy even when systemic features are mild. Further delineation of SRDSA3-associated eye phenotypes can help inform genetic counseling for prognostic estimation of visual loss and disease progression.

JAMA Ophthalmol. doi:10.1001/jamaophthalmol.2017.0046

Published online March 2, 2017.
Author Affiliations: Author affiliations are listed at the end of this article.

Group Information: A complete list of the members of the UK Inherited Retinal Disease Consortium and the 100,000 Genomes Project appears at the end of this article.

Corresponding Author: Graeme C. Black, DPhil, FRCOphth, Manchester Centre for Genomic Medicine, Central Manchester University Hospitals NHS Foundation Trust, MAHSC, Saint Mary's Hospital, Manchester M13 9WL, England (graeme.black @manchester.ac.uk). 
ongenital disorders of glycosylation (CDGs) are a large group of neurometabolic diseases caused by impaired glycoconjugate synthesis. Type I CDGs (CDG-I) result from disruptions in the early $N$-linked glycosylation pathway. ${ }^{1}$ Numerous CDG-I subtypes exist that are characterized by neurologic, developmental, hepatic, and coagulation abnormalities alongside ocular, muscular, skeletal, dermatologic, cardiovascular, or genitourinary involvement in some forms. ${ }^{1,2}$ Approximately 23 different genes have been associated with this group of disorders. ${ }^{1}$ Steroid 5a-reductase type 3 (SRD5A3 [OMIM 611715]) encodes a polyprenol reductase enzyme required for the synthesis of dolichol, the end product of the mevalonate pathway. ${ }^{3}$ Dolichol undergoes phosphorylation to produce dolichol phosphate that serves as the lipid-anchor for $\mathrm{N}$-glycan biosynthesis in the endoplasmic reticulum. ${ }^{3}$

Biallelic mutations in SRD5A3 cause SRD5A3-CDG (formerly known as CDG-Iq [OMIM 612379]), a phenotypically variable form of CDG-I that features nystagmus, optic atrophy, visual loss, muscle hypotonia, intellectual disability, and cerebellar ataxia., ${ }^{3,4}$ Biochemically, SRD5A3-CDG is characterized by a transferrin isoelectric focusing pattern that is typical of CDG-I. ${ }^{5}$ Defective glycan synthesis results in altered sialotransferrin forms, which are detectable by charge differences and characterized by increased disialotransferrin and/or asialotransferrin in cases of CDG-I. ${ }^{5}$ Kahrizi syndrome (OMIM 612713), featuring iris coloboma, juvenile cataract, contractures, kyphosis, mental retardation, motor delay, and lack of speech, has also been reported in association with biallelic variants in SRD5A3. ${ }^{6}$ Patients described thus far have considerable phenotypic overlap with SRD5A3-CDG, although they demonstrate a normal transferrin isoelectric focusing profile. ${ }^{6,7}$ Unlike other CDG-I subtypes, all patients with SRD5A3-CDG develop abnormal ocular phenotypes and almost always experience earlyonset visual loss such that the ocular presentation can be an early and obvious disease-delineating feature.

Previous studies of this disorder focused on genetic findings associated with the neurometabolic and developmental manifestations of the condition, with only 1 study acknowledging a retinal abnormality. ${ }^{8}$ Hence, the appearance, onset, and progression of the SRD5A3-CDG-related retinal phenotype is poorly understood. Our study reports detailed ocular and developmental phenotypes in 7 individuals, from 4 unrelated families with early-onset retinal dystrophy (EORD) who harbor the same SRD5A3 mutation, discovered through wholeexome sequencing (WES) or whole-genome sequencing (WGS).

\section{Methods}

\section{Clinical Assessment}

Study participants were ascertained from Manchester Centre for Genomic Medicine, Manchester, England; Moorfields Eye Hospital, London, England; and St. James's University Hospital, Leeds, England. The Northwest Research Ethics Committee granted approval for all aspects of this study, and the study protocol observed the tenets of the Declaration of Helsinki. ${ }^{9}$ Written informed consent was obtained from each study par-

\section{Key Points}

Question What is the retinal phenotype underlying steroid 5a-reductase type 3 congenital disorder of glycosylation (SRD5A3-CDG)?

Findings This case series of 7 individuals from 4 unrelated families with early-onset retinal dystrophy found, through the use of whole-exome sequencing or whole-genome sequencing, that they harbored an SRD5A3 c.57G>A, p. (Trp19Ter) homozygous mutation, which manifested through early-onset visual loss, nyctalopia, myopia, and nystagmus as well as learning difficulties and psychomotor delay.

Meaning This detailed description of the SRD5A3-CDG retinal phenotype may facilitate disease recognition and prognostic estimation in other cases.

ticipant, or parental informed consent was obtained on behalf of children, as an essential prerequisite for study inclusion. Patient phenotype data were collected over the course of ophthalmic follow-up, spanning a period of 20 years, beginning March 20, 1997, through September 15, 2016.

Each patient underwent full ophthalmic assessment, including visual acuity and dilated fundus examination. Fundus photographs were obtained using conventional $35^{\circ}$ color fundus photography (Topcon Great Britain) or Widefield color fundus imaging (Optos plc). Fundus autofluorescence (FAF) imaging was conducted using either the $55^{\circ}$ Spectralis (Heidelberg Engineering Inc) or ultra-widefield confocal scanning laser imaging (Optos plc). Optical coherence tomography (OCT) was performed using the Spectralis OCT platform. Five patients underwent electroretinography, which was performed using gold-foil electrodes and according to standards specified by the International Society for Clinical Electrophysiology of Vision in 3 patients and using surface electrodes in the other 2 patients. ${ }^{10,11}$ Developmental and dysmorphology assessments were conducted by a clinical geneticist (J.C.S.) or an inherited metabolic disease specialist (S.G.).

\section{Molecular Investigations}

\section{Genetic Analysis}

Targeted next-generation sequencing (105-gene inherited retinal dystrophy panel testing and WES) was conducted, as previously detailed by Arno et al. ${ }^{12}$

The proband of families I and III underwent screening for a panel of 105 known inherited retinal dystrophy genes (described in O'Sullivan et $\mathrm{al}^{13}$ ) at the Manchester Genomic Diagnostic Laboratory. Single-nucleotide polymorphism analysis was performed on family II using an Affymetrix 50k Xba SNP chip (Affymetrix Inc) on DNA samples from the parents, 1 affected child, and 2 unaffected children to identify regions of homozygosity in the affected child for the prioritization of candidate genes. The proband from family IV was screened using a commercially available APEX microarray (Asper Ophthalmics) for 344 published disease-causing variants in 8 genes associated with Lebers congenital amaurosis and EORD. The proband from families I, II, and III underwent WES as part of the UK Inherited Retinal Disease Consortium, an ongoing study of 
inherited retinal disease in families without a molecular diagnosis following targeted gene panel screening.

The affected individual and unaffected parents of family IV underwent WGS as part of the 100,000 Genomes Project. Genomic DNA was processed using a kit (TruSeq DNA PCRFree Sample Preparation; Illumina Inc) and sequenced using a high-throughput sequencing platform (HiSeq X Ten; Illumina Inc), generating minimum coverage of 15 times for more than $97 \%$ of the callable autosomal genome. Readings were aligned to build GRCh37 of the human genome using an aligner (Isaac; Illumina Inc). Single-nucleotide variants and indels (insertions or deletions) were identified using Platypus software (version 0.8.1; Wellcome Trust Centre for Human Genetics) (http://www.well.ox.ac.uk/platypus) and annotated using Cellbase software (https://github.com/opencb/cellbase). Variant filtering was performed using minor allele frequency in publicly available and in-house data sets, predicted protein effect, and familial segregation. Surviving variants were prioritized using 2 prespecified virtual gene panels from PanelApp (https: //bioinfo.extge.co.uk/crowdsourcing/PanelApp/): Intellectual Disability version 1.2, which includes SRD5A3, and Posterior Segment Abnormalities version 1.7. Allelic state was required to match the curated mode of inheritance for variants in panel genes.

The SRD5A3 c.57G>A, p.(Trp19Ter) (GenBank NM _024592) homozygous variant was confirmed by Sanger sequencing using a cycle sequencing kit (BigDye Terminator, version 3.1; Applied Biosystems).

\section{Biochemical Studies}

Where samples were made available, type I $N$-glycosylation defect was confirmed using isoelectric focusing of serum transferrin and blood coagulation studies. ${ }^{5}$

\section{Results}

\section{Patient Phenotypes}

Study participants ( 5 females and 2 males) had a mean (SD) age of 17.1 (3.9) years at their most recent examination, were all of South Asian ethnicity. Phenotypes are summarized in the Table.

\section{Family I}

Family I (G40001), originally from India, had a history of consanguinity and no prior family history of health problems.

The proband, patient I-1, from family I was born slightly underweight at $2.7 \mathrm{~kg}$ and was mildly jaundiced after birth. A developmental and dysmorphology assessment by a clinical geneticist found only mild developmental delay. She walked at age 18 months and developed speech at the normal time. She attended mainstream school where she received assistance because of her visual problems but was able to complete the same level of work as her peers.

At age 5 weeks, she was not fixing and following but was otherwise well. At age 5 years, ophthalmic review identified a decline in visual acuity; fundus imaging and electrophysiological testing led to a preliminary diagnosis of congenital sta- tionary night blindness (Table and Figure $1 \mathrm{~A}$ and $\mathrm{C}$ ). At her latest visit at age 20 years, right and left best-corrected visual acuity measured $1.5 \log$ MAR (20/800 Snellen) with a mild myopic refractive error (Table). Fundus autofluoresence imaging was also abnormal (Figure 1B and D).

Patient I-2 was born at term following an uneventful pregnancy and was otherwise fit and well. At age 5 years, she was described as being hyperactive with an attention deficit and experienced frequent sleep disturbances. At age 7 years, she was examined by a clinical geneticist who diagnosed her as having social communication disorder, behavioral problems, and learning difficulties. Dysmorphology assessment identified her as having thick hair; thick gums; coarse facies; and slender, tapered fingers.

At age 2 months, she presented with multiplanar nystagmus. On examination, she was found to be mildly myopic, while fundoscopy revealed only attenuated retinal blood vessels, and electroretinography demonstrated no recordable response in the dark (Figure $1 \mathrm{E}$ and $\mathrm{G}$ and Table). Fundus autofluoresence at age 3 years was abnormal (Figure $1 \mathrm{~F}$ and $\mathrm{H}$ ). At age 7 years, she began to report symptoms of nyctalopia.

Family II

The proband (II-1) and her affected sister (II-1) from family II (G15567) were born to first-cousin parents of a family who originated from India.

Examination of patient II-1 by a clinical geneticist found she had delayed motor and speech development with associated learning difficulties at a young age. She had variable manifest nystagmus and myopia at age 18 months. At age 5 years, her best-corrected visual acuity measured 3/12 single Kays (0.60 logMAR [20/80 Snellen]) in the right and left eyes (Table). Electrodiagnostic testing at age 11 years identified both rod and cone system dysfunction. In her second decade of life, she became symptomatic with nyctalopia and photophobia. Fundus, FAF, and OCT examinations identified multiple abnormalities indicative of retinitis pigmentosa (RP) (Table and Figure 1I-L and Figure 2A and B).

Patient II-2 was examined by a pediatrician at age 18 months and was found to have normal muscle tone but increased, brisk reflexes and mild hyperkeratosis on the right leg. She also had developmental delay and learning difficulties but had normal growth measurements and a normal skeletal survey. She was particularly anxious and had a very short memory.

The patient was found to have pendular nystagmus and roving eye movements at age 3 months. Electrodiagnostic testing at age 7 years suggested rod and cone dysfunction. By age 15 years, her myopia had increased, and she was experiencing poor night vision and photophobia. Results of fundus, FAF, and OCT examinations were abnormal and indicative of RP in the absence of pigmentary changes (Table and Figures 1M-P and $2 \mathrm{C}$ and $\mathrm{D}$ ).

Family III

The affected sibling pair from family III (LDS3659) were born to apparently nonconsanguineous parents originating from India.

Patient III-1 experienced learning difficulties from a young age and was described as having a slightly "clumsy” walking 


\begin{tabular}{|c|c|c|c|c|c|c|c|}
\hline \multirow[b]{2}{*}{ Characteristic } & \multicolumn{7}{|c|}{ Individual/Sex/Family ID } \\
\hline & I-1/F/G40001.1 & I-2/F/G40001.2 & II-1/F/G15567.1 & II-2/F/G15567.2 & III-1/F/LDS3659.1 & $\begin{array}{l}\mathrm{III}-2 / \mathrm{M} / \\
\text { LDS3659.2 }\end{array}$ & IV-1/M/G15063 \\
\hline \multicolumn{8}{|c|}{ Demographic Characteristics } \\
\hline Ethnicity & South Asian & South Asian & Indian & Indian & Pakistani & Pakistani & Indian \\
\hline Age at onset & 5 wk & $2 \mathrm{mo}$ & $18 \mathrm{mo}$ & $3 \mathrm{mo}$ & $<1 \mathrm{y}$ & $2-3 y$ & $<1 \mathrm{y}$ \\
\hline $\begin{array}{l}\text { Age at last } \\
\text { examination, y }\end{array}$ & 20 & 13 & 18.5 & 14.5 & 16 & 14 & 24 \\
\hline Consanguinity & + & + & + & + & + & - & - \\
\hline \multicolumn{8}{|c|}{ Ophthalmic Findings } \\
\hline $\begin{array}{l}\text { Ophthalmic } \\
\text { history }\end{array}$ & $\begin{array}{l}\text { Failure to fix and } \\
\text { follow, multiplanar } \\
\text { nystagmus, mild } \\
\text { myopia from } 2 \text { mo, } \\
\text { nyctalopia from } \\
6 \text { y, initial } \\
\text { diagnosis of CSNB } \\
\text { made at } 6 \mathrm{y}\end{array}$ & $\begin{array}{l}\text { Multiplanar } \\
\text { nystagmus, } \\
\text { strabismus, } \\
\text { progressive } \\
\text { myopia from } 2 \text { mo, } \\
\text { nyctalopia from } \\
7 \text { y }\end{array}$ & $\begin{array}{l}\text { Variable manifest } \\
\text { nystagmus, squint, } \\
\text { myopia from } \\
18 \text { mo }\end{array}$ & $\begin{array}{l}\text { Nystagmus and } \\
\text { roving eye } \\
\text { movements from } \\
3 \text { mo, myopia, } \\
\text { poor night vision } \\
\text { and photophobia }\end{array}$ & $\begin{array}{l}\text { Roving eye } \\
\text { movements and } \\
\text { nyctalopia from } \\
<1 \text { y, high myopia, } \\
\text { exophoria } \\
\text { decompensating } \\
\text { into an exotropia } \\
\text { from } 16 \text { y, central } \\
\text { scotomata }\end{array}$ & $\begin{array}{l}\text { Roving eye } \\
\text { movements from } \\
2-3 \text { y, nyctalopia, } \\
\text { high myopia, } \\
\text { exophoria }\end{array}$ & $\begin{array}{l}\text { Early-onset } \\
\text { nystagmus and } \\
\text { myopia }\end{array}$ \\
\hline $\begin{array}{l}\text { BCVA (Snellen } \\
\text { equivalent) } \\
{[\text { age, y] }}\end{array}$ & $\begin{array}{l}1.5 \log M A R \\
(20 / 640) \text { OU [20] }\end{array}$ & $\begin{array}{l}1.3 \text { logMAR } \\
(20 / 400) \text { OU [7]; } \\
1.04 \text { logMAR } \\
(20 / 250) \text { OD ; } \\
1.20 \text { logMAR } \\
(20 / 320) \text { OS [13] }\end{array}$ & $\begin{array}{l}\text { 0.900 Crowded } \\
\text { logMAR }(20 / 160) \\
\text { OD; } \\
0.800(20 / 125) \\
\text { crowded logMAR } \\
\text { OS [6]; } 0.72 \\
\text { logMAR (20/100) } \\
\text { OD; 0.36 logMAR } \\
(20 / 50) \text { OS [18.5] }\end{array}$ & $\begin{array}{l}1.0 \text { logMAR } \\
(20 / 200) \text { OD; } 0.8 \\
\text { logMAR }(20 / 125) \\
\text { OS }[15]\end{array}$ & NA & NA & $\begin{array}{l}0.6 \text { logMAR } \\
(20 / 80) \text { OU [4.5]; } \\
1.0 \text { logMAR } \\
(20 / 200) \text { OU } \\
{[24 \mathrm{y}]}\end{array}$ \\
\hline $\begin{array}{l}\text { Refractive } \\
\text { error (age) }\end{array}$ & $\begin{array}{l}-1.00 /+0.25 \times 90 \\
\text { OD; } \\
-1.25 /+0.25 \times 80 \\
\text { OS }(20 y)\end{array}$ & $\begin{array}{l}-2.00 /+1.00 \\
\times 100 \mathrm{OD} ; \\
-3.00 /+1.00 \times 80 \\
\text { OS }(2 \mathrm{mo}) ; \\
-6.00 /+1.75 \times 90 \\
\text { OD and } \\
-6.50 /+1.00 \times 90 \\
\text { OS }(3 \mathrm{y})\end{array}$ & $\begin{array}{l}-2.5 /-2.5 \times 180 \\
\text { OD; } \\
-1.5 /-3.0 \times 170 \\
\text { OS }(6 y) ; \\
-3.00 /-3.5 \times 180 \\
\text { OD; } \\
-3.50 /-4.0 \times 160 \\
\text { OS }(18.5 y)\end{array}$ & $\begin{array}{l}-1.5 /-1.25 \times 180 \\
\text { OD; } \\
-2.00 /-2.00 \\
\times 180 \text { OS }(18 \mathrm{mo}) \\
-5.5 /-3.75 \times 155 \\
\text { OD; } \\
-5.5 /-3.75 \times 100 \\
\text { OS }(15 \mathrm{y})\end{array}$ & $\begin{array}{l}-15.50 /+0.25 \\
\times 109 \text { OD; } \\
-14.00 / \\
+1.00 \times 92 \text { os } \\
(16 y)\end{array}$ & $\begin{array}{l}-9.50 /+1.50 \\
\times 103 \mathrm{OD} \\
-8.25 /+2.5 \times 106 \\
\text { OS }(14 \mathrm{y})\end{array}$ & $\begin{array}{l}\text { OD: } \\
-7.00 /-0.75 \\
\times 180 ; \text { OS: }-7.5 \\
\text { DS }(24 \mathrm{y})\end{array}$ \\
\hline $\begin{array}{l}\text { Fundus } \\
\text { imaging }\end{array}$ & $\begin{array}{l}\text { Optic disc pallor, } \\
\text { foveal hypoplasia, } \\
\text { granular } \\
\text { appearance of } \\
\text { peripheral retina, } \\
\text { attenuated retinal } \\
\text { vasculature }\end{array}$ & $\begin{array}{l}\text { Subtle temporal } \\
\text { optic disc pallor, } \\
\text { mildly attenuated } \\
\text { retinal arterioles, } \\
\text { prominent nerve } \\
\text { fiber layer visibly } \\
\text { radiating around } \\
\text { the superior and } \\
\text { inferior vascular } \\
\text { arcades; patchy } \\
\text { (RE) and stippled } \\
\text { (LE) macular } \\
\text { reflex }\end{array}$ & $\begin{array}{l}\text { Tilted optic disc } \\
\text { with temporal } \\
\text { pallor, } \\
\text { peripapillary } \\
\text { atrophy } \\
\text { temporally, } \\
\text { absence of foveal } \\
\text { reflex (LE only), } \\
\text { attenuated retinal } \\
\text { vasculature }\end{array}$ & $\begin{array}{l}\text { Myopic tilted discs, } \\
\text { attenuated retinal } \\
\text { vasculature, subtle } \\
\text { mottling in the } \\
\text { retinal periphery }\end{array}$ & $\begin{array}{l}\text { Myopic tilted discs, } \\
\text { attenuated retinal } \\
\text { vasculature, subtle } \\
\text { mottling in the } \\
\text { retinal periphery }\end{array}$ & $\begin{array}{l}\text { Myopic tilted discs, } \\
\text { attenuated retinal } \\
\text { vasculature, subtle } \\
\text { mottling in the } \\
\text { retinal periphery }\end{array}$ & $\begin{array}{l}\text { Optic disc pallor, } \\
\text { attenuated } \\
\text { retinal } \\
\text { vasculature }\end{array}$ \\
\hline FAF & $\begin{array}{l}\text { Well-defined } \\
\text { ring of } \\
\text { hyperautofluores- } \\
\text { cence around the } \\
\text { macula }\end{array}$ & $\begin{array}{l}\text { Well-defined } \\
\text { ring of } \\
\text { hyperautofluores- } \\
\text { cence around the } \\
\text { macula }\end{array}$ & $\begin{array}{l}\text { Well-defined } \\
\text { ring of } \\
\text { hyperautofluores- } \\
\text { cence around the } \\
\text { macula }\end{array}$ & $\begin{array}{l}\text { Diffuse ring of } \\
\text { hyperautofluores- } \\
\text { cence around the } \\
\text { macula }\end{array}$ & $\begin{array}{l}\text { Diffuse ring of } \\
\text { hyperautofluores- } \\
\text { cence around the } \\
\text { macula }\end{array}$ & $\begin{array}{l}\text { Well-defined } \\
\text { ring of } \\
\text { hyperautofluores- } \\
\text { cence around the } \\
\text { macula }\end{array}$ & $\begin{array}{l}\text { Diffuse ring of } \\
\text { hyperautofluores- } \\
\text { cence around the } \\
\text { macula }\end{array}$ \\
\hline OCT & Data not available & Data not available & $\begin{array}{l}\text { Widespread loss } \\
\text { of outer retinal } \\
\text { structures with } \\
\text { relative } \\
\text { preservation of } \\
\text { foveal structures, } \\
\text { including } \\
\text { photoreceptors }\end{array}$ & $\begin{array}{l}\text { Widespread loss } \\
\text { of outer retinal } \\
\text { structures with } \\
\text { relative } \\
\text { preservation of } \\
\text { foveal structures, } \\
\text { including } \\
\text { photoreceptors }\end{array}$ & $\begin{array}{l}\text { Widespread loss } \\
\text { of outer retinal } \\
\text { structures and } \\
\text { complete absence } \\
\text { of the } \\
\text { photoreceptor } \\
\text { layer }\end{array}$ & $\begin{array}{l}\text { Widespread loss } \\
\text { of outer retinal } \\
\text { structures with } \\
\text { relative } \\
\text { preservation of } \\
\text { foveal structures, } \\
\text { including } \\
\text { photoreceptors }\end{array}$ & $\begin{array}{l}\text { Widespread loss } \\
\text { of outer retinal } \\
\text { structures with } \\
\text { relative } \\
\text { preservation of } \\
\text { foveal structures, } \\
\text { including } \\
\text { photoreceptors }\end{array}$ \\
\hline $\begin{array}{l}\text { ERG (age } \\
\text { at testing) }\end{array}$ & $\begin{array}{l}\text { Indicative of } \\
\text { rod-cone } \\
\text { dystrophy (no } \\
\text { details available) } \\
(5 \mathrm{y})\end{array}$ & $\begin{array}{l}\text { Low-amplitude } \\
\text { light-adapted } \\
\text { response, } \\
\text { extinguished } \\
\text { dark-adapted } \\
\text { response ( } 2 \text { mo) }\end{array}$ & $\begin{array}{l}\text { Undetectable } \\
\text { rod-specific } \\
\text { responses, delayed } \\
\text { and subnormal } \\
\text { cone-specific } \\
\text { responses }(11 \mathrm{y})\end{array}$ & $\begin{array}{l}\text { Limited } \\
\text { compliance with } \\
\text { test but reduced } \\
\text { and delayed } \\
\text { cone-specific } \\
\text { responses found } \\
\text { with rod } \\
\text { involvement }\end{array}$ & NA & NA & $\begin{array}{l}\text { Profoundly } \\
\text { electronegative } \\
\text { ERG, grossly } \\
\text { delayed } \\
\text { cone-specific } \\
\text { responses }\end{array}$ \\
\hline
\end{tabular}

style. Her family noticed she had poor visual behavior within the first year of life. A myopic refractive error was detected at age 18 months, which progressed to high myopia by age 16 years
(Table). Examination of the fundus, by color and FAF imaging, revealed abnormalities suggestive of retinal pigment epithelium malfunction (Table and Figure 1T). The OCT scans were 


\begin{tabular}{|c|c|c|c|c|c|c|c|}
\hline \multirow[b]{2}{*}{ Characteristic } & \multicolumn{7}{|c|}{ Individual/Sex/Family ID } \\
\hline & I-1/F/G40001.1 & I-2/F/G40001.2 & II-1/F/G15567.1 & II-2/F/G15567.2 & III-1/F/LDS3659.1 & $\begin{array}{l}\text { III-2/M/ } \\
\text { LDS3659.2 }\end{array}$ & IV-1/M/G15063 \\
\hline $\begin{array}{l}\text { Developmental/ } \\
\text { neurological } \\
\text { findings }\end{array}$ & $\begin{array}{l}\text { Mild } \\
\text { developmental } \\
\text { delay up to age } 5 \text { y }\end{array}$ & $\begin{array}{l}\text { Dysmorphic, } \\
\text { communication } \\
\text { and behavioral } \\
\text { problems, learning } \\
\text { difficulties, } \\
\text { recurrent } \\
\text { respiratory } \\
\text { infections, gait } \\
\text { ataxia }\end{array}$ & $\begin{array}{l}\text { Psychomotor } \\
\text { delay, learning } \\
\text { difficulties }\end{array}$ & $\begin{array}{l}\text { Increased brisk } \\
\text { reflexes, } \\
\text { psychomotor } \\
\text { delay, and learning } \\
\text { difficulties }\end{array}$ & $\begin{array}{l}\text { Learning } \\
\text { difficulties, gait } \\
\text { ataxia, normal } \\
\text { reflexes, mild } \\
\text { upper-limb } \\
\text { coordination } \\
\text { difficulties on } \\
\text { finger-nose test; } \\
\text { normal height, } \\
\text { weight, and head } \\
\text { circumference }\end{array}$ & $\begin{array}{l}\text { Learning } \\
\text { difficulties, } \\
\text { developmental } \\
\text { delay, gait ataxia, } \\
\text { normal reflexes, } \\
\text { mild upper-limb } \\
\text { coordination } \\
\text { difficulties on } \\
\text { finger-nose test; } \\
\text { recurrent } \\
\text { respiratory } \\
\text { infections; normal } \\
\text { height, weight, } \\
\text { and head } \\
\text { circumference }\end{array}$ & $\begin{array}{l}\text { Developmental } \\
\text { delay, learning } \\
\text { difficulties, } \\
\text { scoliosis }\end{array}$ \\
\hline $\begin{array}{l}\text { Other } \\
\text { investigations }\end{array}$ & $\begin{array}{l}\text { Normal urine } \\
\text { organic acid and } \\
\text { plasma phytanic } \\
\text { acid levels }\end{array}$ & $\begin{array}{l}\text { Normal hearing } \\
\text { assessment results; } \\
\text { normal values for } \\
\text { uMPS, } \\
\text { oligosaccharides, } \\
\text { and lysosomal } \\
\text { enzymes; normal } \\
\text { radiographic } \\
\text { findings and aCGH } \\
\text { results }\end{array}$ & $\begin{array}{l}\text { Normal values for } \\
\text { VLCFAs, lysosomal } \\
\text { enzymes, and } \\
\text { white cell and } \\
\text { plasma enzymes }\end{array}$ & $\begin{array}{l}\text { Normal values for } \\
\text { VLCFAs, lysosomal } \\
\text { enzymes, and } \\
\text { white cell and } \\
\text { plasma enzymes }\end{array}$ & & & \\
\hline \multicolumn{4}{|c|}{$\begin{array}{l}\text { Abbreviations: aCGH, array comparative genomic hybridization; DS, diopter } \\
\text { sphere; ERG, electroretinography; FAF, fundus autofluoresence; NA, not } \\
\text { available; OCT, optical coherence tomography; OD, right eye; OS, left eye; }\end{array}$} & \multicolumn{4}{|c|}{$\begin{array}{l}\text { OU, both eyes; uMPS, urine mucoplysaccharides; VLCFAs, very long-chain fatty } \\
\text { acids; +, present; -, absent. }\end{array}$} \\
\hline
\end{tabular}

corroborative of this finding and indicated loss of outer segment structures with complete loss of the photoreceptor layer (Figure 2E).

Patient III-2, when examined at age 14 years, was found to have an ataxic gait and reduced upper-limb coordination, both of which are signs of mild cerebellar disease. He also demonstrated global developmental delay and experienced recurrent respiratory tract infections.

He experienced loss of vision with associated roving eye movements between ages 2 and 3 years. He also presented with early-onset nyctalopia and high myopia (Table). Ophthalmic examination revealed abnormalities similar to those of his brother, apart from a small region of photoreceptor preservation within the central macula (Table and Figure 2F).

\section{Family IV}

The proband (IV-1) from family IV (G15063), a male, was born to apparently nonconsanguineous parents originating from India. Examination of patient IV- 1 at age $4 \frac{1}{2}$ years identified developmental delay, learning difficulties, and abnormal curvature of the spine (Table). His ophthalmic history revealed infantile-onset nystagmus and reduced vision. At age $4 \frac{1}{2}$ years, he was found to have reduced visual acuity (0.60 logMAR OU [20/80 Snellen]) and myopia (Table). At his most recent examination at age 24 years, his vision had deteriorated (Table), and fundus examination revealed retinal vessel attenuation and pale optic discs (Figure $1 \mathrm{Q}$ and S). Electroretinography indicated both rod and cone dysfunction (Table), and an OCT scan revealed loss of outer segment structures with relative preservation of the central macula, bilaterally (Figure 2G and $\mathrm{H}$ ).

\section{Molecular Analysis}

Clinically available genetic testing did not identify any potentially pathogenic variants in 105 known retinal dystrophy genes in the proband of families I and III. Autozygosity mapping and candidate gene sequencing did not identify any pathogenic variants in the proband of family II. Apex array analysis in patient IV-1 was also mutation negative. Subsequent WES or WGS led to the identification of SRD5A3 c.57G >A, p.(Trp19Ter) homozygous variant in each proband. Sanger sequencing confirmed the presence and zygosity of this variant in every affected member of each family. The SRD5A3 c.57G>A, p.(Trp19Ter) variant has an allele frequency of 0.001174 in 4684 control individuals of South Asian ethnicity, according to the Exome Aggregation Consortium data set (http://exac.broadinstitute.org/). In the homozygous state, this same variant has been described as the cause of SRD5A3-CDG in 4 unrelated families. ${ }^{4,8,14}$

Both siblings from family III underwent screening for biochemical abnormalities that may be associated with CDG. ${ }^{5}$ Mild abnormalities of blood clotting (activated partial thromboplastin time [aPTT], 43.6 seconds; aPTT ratio, 1.4 [international normalized ratio, 3.23]) and a microcytic hypochromic blood profile were observed in both. Liver function test results were normal; however, a CDG type I pattern of transferrin glycoforms was observed.

\section{Discussion}

Biallelic mutations in SRD5A3 are associated with SRD5A3CDG, a phenotypically variable disorder of $N$-linked glycosyla- 
Figure 1. Color Fundus and Fundus Autofluorescence Images of Patients With SRD5A3 c.57G>A, p.(Trp19Ter) Variant
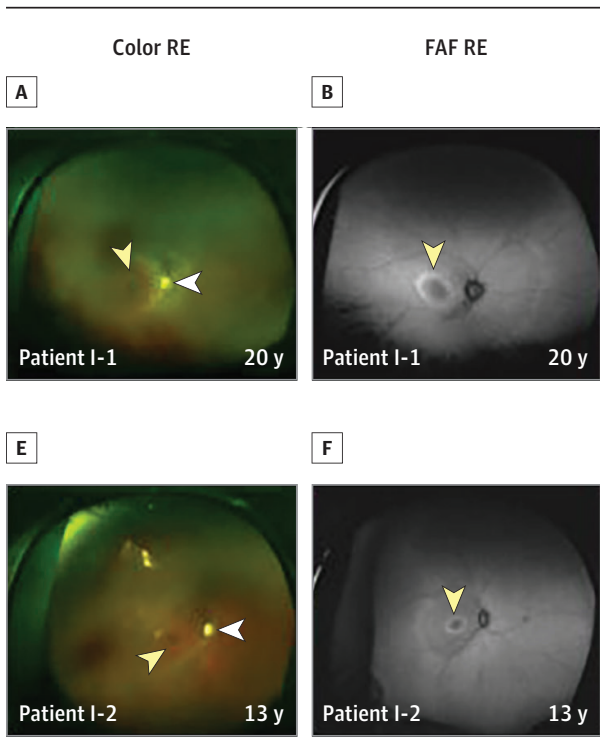

$\mathbf{G}$

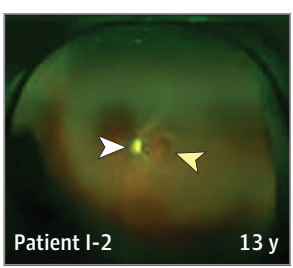

$\mathbf{K}$
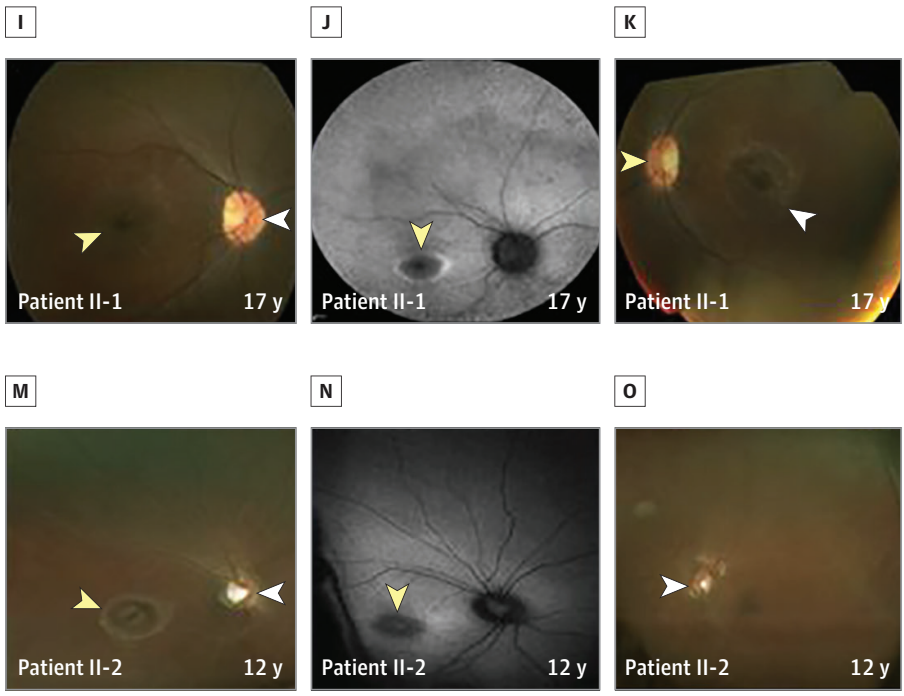

$\mathbf{N}$

0
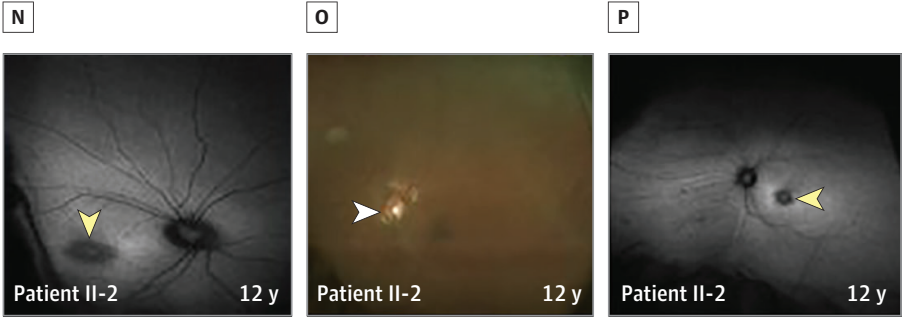

Q

$\mathbf{R}$
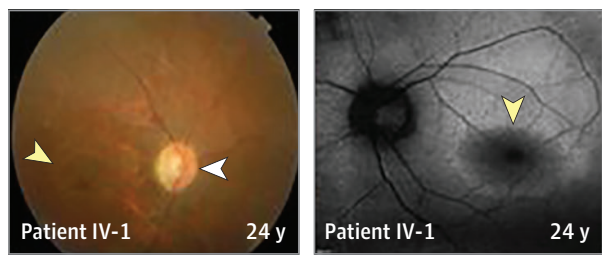

$\mathbf{S}$

$\mathbf{T}$

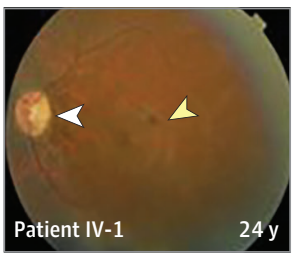

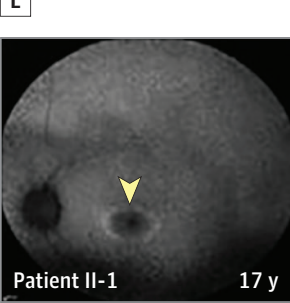

D

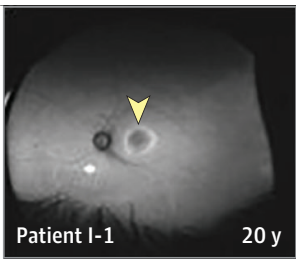

H

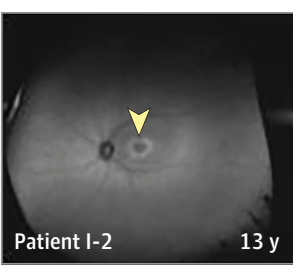

$\mathrm{L}$

Patient II-1

$17 y$ tion that is normally characterized by neurodevelopmental abnormalities and ophthalmic manifestations. ${ }^{3,4}$ We describe 7 patients from 4 families with a retinopathy consequent on the SRD5A3 c.57G>A, p.(Trp19Ter) mutation. This mutation has been reported previously to cause SRD5A3-CDG in 4 other unrelated families. ${ }^{4,8,14}$

Our case series provides an in-depth description of the ocular symptomology and appearance over the course of ophthalmic follow-up. The retinopathy, unlike the extraocular fea- tures of this disease, appears to be slowly progressive. On fundal view, signs of retinal disease may be subtle and bone spicules may be absent in young patients. Similarly, syndromic manifestations associated with SRD5A3 mutation may also be mild. This detailed description of retinal phenotype could be important for early disease recognition because it appears to be a consistent primary presenting feature. Earlyonset visual loss ( $\leq 3$ years of age; mean [SD] best-corrected visual acuity, +0.95 [0.34] logMAR [20/180 Snellen]) and 
Figure 2. Optical Coherence Tomography in Patients With SRD5A3 c.57G>A, p.(Trp19Ter) Variant

A Patient II-1 RE, $17 \mathrm{y}$

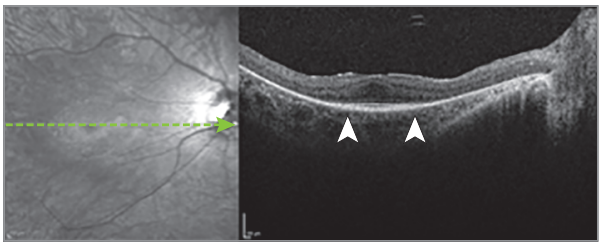

B Patient II-1 LE, 17 y

C Patient II-2 RE, 12 y

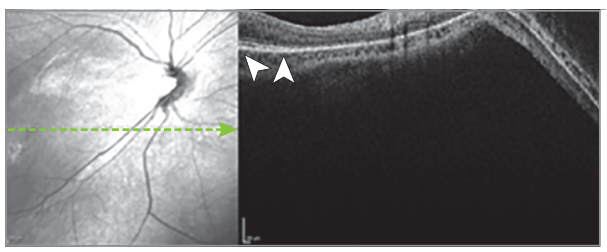

D Patient II-2 LE, 12 y

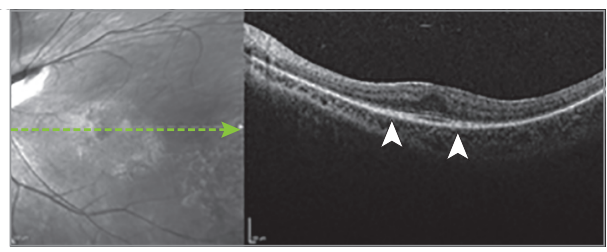

E Patient III-1 RE, $14 \mathrm{y}$

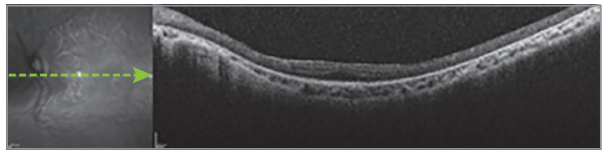

F Patient III-2 RE, $12 \mathrm{y}$

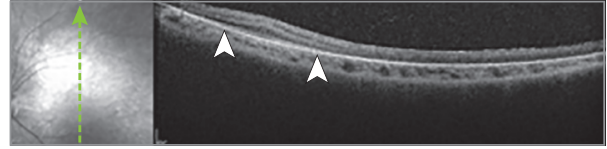

G Patient IV-1 RE, $24 \mathrm{y}$

H Patient IV-1 LE, 24 y
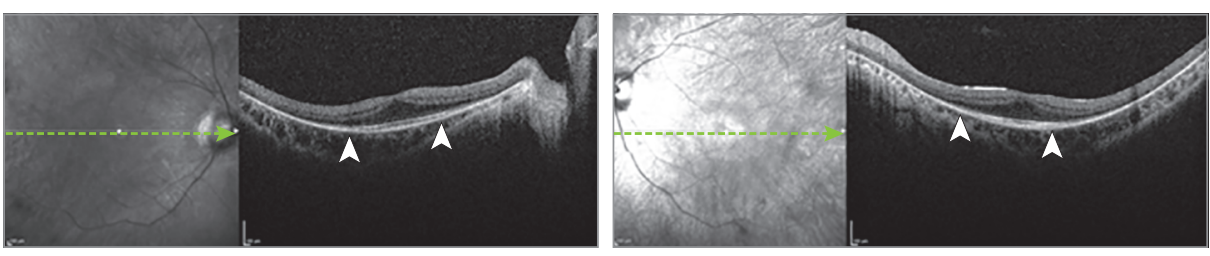

Optical coherence tomographic scans shown as horizontal (A through $E, G$, and $\mathrm{H}$ ) or vertical (F) scans and accompanying en face infrared image with location at which the scan through the macula was taken (indicated by green line). Arrowheads demarcate the transition of absent or present photoreceptors (except in C, where part of the macula is not visible, and in $\mathrm{E}$, where the photoreceptor layer is completely absent). LE indicates left eye; $R E$, right eye. nystagmus are consistent manifestations associated with the SRD5A3 c.57G>A, p. (Trp19Ter) variant in this cohort of 7 patients. Other shared ocular findings were retinal arteriolar attenuation in the absence of bone spicule formation ( $n=7)$, childhood-onset nyctalopia ( $n=5$ ), and optic disc pallor $(n=5)$. In addition, each of the patients described in this series experienced varying degrees of progressive myopia (mean [SD] refractive error, -6.71 [-4.22]), ranging from relatively mild to high (Table). None of the patients were microphthalmic, and they did not have ocular colobomata as has been described in association with other SRD5A3 mutations. ${ }^{4}$ Mutual systemic associations included learning difficulties and developmental delay. One patient was found to have only mild developmental delay as a young child ( $<5$ years), which may have been attributable to her severe visual impairment because she went on to meet normal developmental and intellectual milestones with increasing age.

Despite the absence of a pigmentary retinopathy, widespread loss of outer retinal structures was evidenced by OCT, with relative preservation of foveal photoreceptors and only mild epiretinal membrane formation (Figure 2A-H). Electroretinography, when performed $(n=5)$, identified dysfunction in both rod and cone pathways at the level of the photoreceptor, allowing discrimination from disorders involving the photoreceptor-bipolar cell synapse, such as congenital stationary night blindness, as 3 of the 7 patients initially received a clinical diagnosis of congenital stationary night blindness. Previous reports of patients with $S R D 5 A 3$ mutations have not described OCT findings. There has been a single description of retinal bone spicule pigmentation in an adult sibling pair with the SRD5A3 c.57G>A, p.(Trp19Ter) variant. Because of the lack of previous descriptions of RP as a feature of SRD5A3-CDG, Kara and colleagues ${ }^{8}$ hypothesized that RP may be a late-onset feature of the condition. Our findings suggest that the onset of retinal degeneration is likely to occur in childhood in at least a proportion of cases. Ocular imaging and FAF suggest early dysfunction of the retinal pigment epithelium.

Rhodopsin is a pigment-containing, G protein-coupled receptor that is expressed in rod photoreceptors cells, where it specifically localizes to the rod outer segments. ${ }^{15}$ Studies have shown that the $\mathrm{N}$-terminus of rhodopsin contains $2 \mathrm{~N}$-linked glycosylation sequences. ${ }^{16}$ Mutations at glycosylated amino acid residues or surrounding glycosylation consensus sequences of rhodopsin cause autosomal dominant and sectoral RP in humans. ${ }^{17,18}$ Studies in animal models expressing nonglycosylated rhodopsin have shown that, although the mutant proteins undergo normal biosynthesis, folding, and trafficking, they confer toxicity, which causes rod cell death and leads 
to light-sensitive retinal degeneration. ${ }^{19}$ Evidence of whether nonglycosylated rhodopsin incorporates into and initiates disc morphogenesis in rod outer segments is conflicting. ${ }^{20,21}$ It is possible that the $S R D 5 A 3$ c.57G >A, p.(Trp19Ter) variant prevents normal glycosylation of rhodopsin in the retina and subsequently impairs its normal incorporation and/or function in the rod outer segments, thereby leading to defective phototransduction and loss of vision before the eventual photoreceptor death and the presentation of RP. Similarly, nonglycosylation of other retinal proteins, such as ABCA4, known to have $7 \mathrm{~N}$-glycosylation sites, could also lead to defective phototransduction and eventual cell death. ${ }^{22}$ This is an area that warrants further research.

The SRD5A3 c.57G>A, p.(Trp19Ter) variant has an allele frequency of 0.0012 in the South Asian population according to the Exome Aggregation Consortium data set. This frequency is 30 times higher than for other ethnic groups, suggesting that SRD5A3 c.57G >A, p.(Trp19Ter) is an ancestral variant within this specific population. Further, findings from our cohort suggest that phenotypic subtleties mean this condition goes unrecognized or unsuspected. Alongside recent evidence for a role of other glycosylation disorder genes in nonsyndromic retinal dystrophy ( $P O M G N T 1^{23}$ and $D H D D S^{24}$ ), we suggest that CDG genes be considered in clinical diagnostic gene panels for retinal disease.

\section{Limitations}

For some of the families described in this study, parental DNA samples were unavailable to confirm zygosity of the SRD5A3 c.57G>A, p.(Trp19Ter) homozygous variant identified in each affected family member. Therefore, we cannot definitively exclude the possibility of a heterozygous deletion on 1 allele. Fur- thermore, because of the limited information available regarding each family pedigree, we cannot exclude the possibility that each of the 5 families described here shares a distant, common ancestor.

\section{Conclusions}

This case series provides a detailed account of the retinal dystrophy consequent on the $S R D 5 A 3$ c.57G >A, p.(Trp19Ter) mutation, delineating the complex phenotype associated with SRD5A3-CDG. Furthermore, we illustrate the wide variability in onset and progression of the disorder in patients with the same null mutation. We report EORD as a novel feature of SRD5A3-CDG and suggest that retinal degeneration without pigmentary change may be an early manifestation of CDG that may progress to RP over time. Of note, our findings also suggest that SRD5A3 may cause these ocular manifestations alongside mild learning difficulties in some instances, in contrast to the neurodevelopmental delay and other systemic features usually associated with SRD5A3-CDG. ${ }^{3,4}$ Our work adds to cumulative evidence that next-generation sequencing offers a proficient means of diagnosis for this genetically heterogeneous and phenotypically variable group of conditions. ${ }^{6,25,26}$ For CDG, precise diagnosis enables the provision of more accurate prognostic information regarding loss of vision and risk of later-onset manifestations. Better understanding of the pathogenesis of SRD5A3-mediated retinal disease could lead to the development of novel therapeutic strategies. Findings in our cohort show that the macula, although nonfunctional, remains structurally intact, potentially contributing to making this condition a good target for gene therapy.

\section{ARTICLE INFORMATION}

Accepted for Publication: January 8, 2017.

Published Online: March 2, 2017.

doi:10.1001/jamaophthalmol.2017.0046

Author Affiliations: Genomic Medicine, Division of Evolution and Genomic Sciences, Faculty of Biology, Medicines and Health, University of Manchester. Manchester Academic Health Science Centre (MAHSC), Manchester, England (Taylor, Clayton-Smith, Black); Manchester Centre for Genomic Medicine, Central Manchester University Hospitals NHS Foundation Trust, MAHSC, Saint Mary's Hospital, Manchester, England (Taylor, Clayton-Smith, Black); UCL Institute of Ophthalmology, University College London, London, England (Arno, Hull, Michaelides, Moore Hardcastle, Webster); Section of Ophthalmology and Neuroscience, Leeds Institute of Biomedical and Clinical Sciences, University of Leeds, Leeds, England (Poulter, Khan, Ali, Toomes, McKibbin, Inglehearn); Department of Ophthalmology, St. James's University Hospital, Leeds, England (Khan, McKibbin); Manchester Royal Eye Hospital, Manchester Academic Health Science Centre, Central Manchester Foundation Trust, Manchester, England (Morarji); Moorfields Eye Hospital, London, England (Hull, Michaelides, Hardcastle, Webster); UCL Genetics Institute, University College London, London, England (Pontikos); Genomics England, Queen Mary University of London, London,
England (Rueda Martin, Smith); Metabolic Unit Great Ormond Street Hospital, London, England (Grunewald); Institute for Child Health, University College London, London, England (Grunewald); Department of Ophthalmology, University of California, San Francisco, Medical School, San Francisco (Moore).

Author Contributions: Dr Taylor and Prof Black had full access to all the data in the study and take responsibility for the integrity of the data and accuracy of the data analysis.

Study concept and design: Ali, Toomes, Clayton-Smith, Michaelides, Moore, Inglehearn, Black.

Acquisition, analysis, or interpretation of data Taylor, Arno, Poulter, Khan, Morarji, Hull, Pontikos Rueda Martin, Smith, McKibbin, Clayton-Smith, Michaelides, Moore, Hardcastle, Webster. Drafting of the manuscript: Taylor, Smith, Clayton-Smith, Michaelides, Black.

Critical revision of the manuscript for important intellectual content: Taylor, Arno, Poulter, Khan, Morarji, Hull, Pontikos, Rueda Martin, Ali, Toomes, McKibbin, Clayton-Smith, Grunewald, Michaelides, Moore, Hardcastle, Inglehearn, Webster. Statistical analysis: Khan, Pontikos, Rueda Martin, Black.

Obtained funding: Ali, Toomes, Michaelides, Moore, Hardcastle, Inglehearn, Webster, Black. Administrative, technical, or material support:
Taylor, Poulter, Toomes, Grunewald, Michaelides، Inglehearn, Webster, Black. Study supervision: Toomes, McKibbin, Clayton-Smith, Moore, Inglehearn, Black.

Conflict of Interest Disclosures: All authors have completed and submitted the ICMJE Form for Disclosure of Potential Conflicts of Interest and none were reported.

Funding/Support: This work was funded by RP Fighting Blindness and Fight for Sight (RP Genome Project GR586) and Rosetrees Trust, Fight for Sight (family II), Moorfields Eye Hospital Special Trustees, National Institute for Health Research (NIHR) Biomedical Research Centre at Moorfields Eye Hospital, National Health Service (NHS) Foundation Trust, and UCL Institute of Ophthalmology (Drs Khan, Webster, and Hardcastle). Dr Khan is supported by a National Institute for Health Research Rare Diseases Translational Research Collaboration fellowship award.

Role of the Funder/Sponsor: The funding sources had no role in design and conduct of the study; collection, management, analysis, and interpretation of the data; preparation, review, or approval of the manuscript; and decision to submit the manuscript for publication.

Group Information: Members of the UK Inherited Retinal Disease Consortium include Graeme C. Black, DPhil, FRCOphth (study chair); Georgina Hall, 
MSc; Stuart Ingram, BSc; Rachel L. Taylor, PhD; Simon Ramsden, PhD; Forbes Manson, PhD Panagiotis Sergouniotis, PhD; Andrew R. Webster, MD(Res), FRCOphth; Alison J. Hardcastle, PhD; Michel Michaelides, MD(Res), FRCOphth; Vincent Plagnol, PhD; Nikolas Pontikos, PhD; Michael Cheetham, PhD; Gavin Arno, PhD; Alessia Fiorentino, PhD; Chris F. Inglehearn, PhD; Carmel Toomes, PhD; Manir Ali, PhD; Martin McKibbin, FRCOphth; James A. Poulter, PhD; Kamron N. Khan, PhD, FRCOphth; Emma Lord, MSc; Andrea Nemeth, PhD, FRCP; Susan Downes, MD, FRCOphth; Jing Yu, PhD; Stefano Lise, PhD; and Veronica van Heyningen, PhD. Members of the 100,000 Genomes Project include Mark Caulfield, MBBS, MRCP, MD, FRCP, FAHA, FBHS, FMedSci, FESC, FBPS; Andrew Devereau, PhD; Clare Turnbull, MD, PhD, MA, MSc, MRCP; Dina Halai, MSc; Ellen Thomas, MBBS, PhD; Emma Baple, MBBS, PhD; Freya Boardman-Pretty, PhD; Katarzyna Witkowska, PhD; Laura Riley, MSc; Louise Jones, PhD; Mark Bale, PhD; Matina Prapa, PhD; Nirupa Murugaesu, PhD, MRCP; Richard Scott, MBBS, PhD; Simon Thompson, PhD; Tim Hubbard, PhD; Tom Fowler, PhD; Augusto Rendon, PhD; Alona Sosinsky, PhD; Antonio Rueda Martin, PhD; Damian Smedley, PhD; Ellen McDonagh, PhD; and Katherine Smith, PhD.

Disclaimer: The views expressed are those of the authors and not necessarily those of the NHS, NIHR, or Department of Health.

Additional Contributions: The authors acknowledge the support of the Manchester Academic Health Science Centre and the Manchester National Institute for Health Research Biomedical Research Centre. This research was made possible through access to the data and findings generated by the 100,000 Genomes Project. The 100,000 Genomes Project is managed by Genomics England Limited (a wholly owned company of the Department of Health) and is funded by the NIHR and NHS England. The Wellcome Trust, Cancer Research UK, and the Medical Research Council also funded the research infrastructure. The authors also acknowledge Genomics England and the Ophthalmology Genomics England Clinical Interpretation Partnership for enabling this research. We thank the families for agreeing to participate in this study.

\section{REFERENCES}

1. Cylwik B, Naklicki M, Chrostek L, Gruszewska E. Congenital disorders of glycosylation, part I: defects of protein N-glycosylation. Acta Biochim Pol. 2013; 60(2):151-161.
2. Jaeken J, Matthijs G. Congenital disorders of glycosylation: a rapidly expanding disease family. Annu Rev Genomics Hum Genet. 2007;8:261-278.

3. Cantagrel V, Lefeber DJ, $\mathrm{Ng} \mathrm{BG}$, et al. SRD5A3 is required for converting polyprenol to dolichol and is mutated in a congenital glycosylation disorder. Cell. 2010;142(2):203-217.

4. Morava E, Wevers RA, Cantagrel V, et al. A novel cerebello-ocular syndrome with abnormal glycosylation due to abnormalities in dolicho metabolism. Brain. 2010;133(11):3210-3220.

5. Lefeber DJ, Morava E, Jaeken J. How to find and diagnose a CDG due to defective $\mathrm{N}$-glycosylation. J Inherit Metab Dis. 2011;34(4):849-852.

6. Kahrizi K, Hu CH, Garshasbi M, et al. Next generation sequencing in a family with autosomal recessive Kahrizi syndrome (OMIM 612713) reveals a homozygous frameshift mutation in SRD5A3. Eur J Hum Genet. 2011;19(1):115-117.

7. Al-Gazali L, Hertecant J, Algawi K, El Teraifi H, Dattani M. A new autosomal recessive syndrome of ocular colobomas, ichthyosis, brain malformations and endocrine abnormalities in an inbred Emirati family. Am J Med Genet A. 2008;146A(7):813-819.

8. Kara B, Ayhan Ö, Gökçay G, Başboğaoğlu N, Tolun A. Adult phenotype and further phenotypic variability in SRD5A3-CDG. BMC Med Genet. 2014; 15:10.

9. World Medical Association. World Medical Association Declaration of Helsinki: ethical principles for medical research involving human subjects. JAMA. 2013;310(20):2191-2194. doi:10 .1001/jama.2013.281053

10. McCulloch DL, Marmor MF, Brigell MG, et al. ISCEV Standard for full-field clinical electroretinography (2015 update). Doc Ophthalmol. 2015;130(1):1-12.

11. Bach $M$, Brigell MG, Hawlina M, et al. ISCEV standard for clinical pattern electroretinography (PERG): 2012 update. Doc Ophthalmol. 2013;126(1): 1-7.

12. Arno G, Holder GE, Chakarova C, et al; UK Inherited Retinal Disease Consortium. Recessive retinopathy consequent on mutant G-protein $\beta$ subunit 3 (GNB3). JAMA Ophthalmol. 2016;134(8): 924-927.

13. O'Sullivan J, Mullaney BG, Bhaskar SS, et al A paradigm shift in the delivery of services for diagnosis of inherited retinal disease. J Med Genet. 2012;49(5):322-326

14. Gründahl JE, Guan Z, Rust S, et al. Life with too much polyprenol: polyprenol reductase deficiency. Mol Genet Metab. 2012;105(4):642-651.
15. Palczewski K. G protein-coupled receptor rhodopsin. Annu Rev Biochem. 2006;75:743-767.

16. Hargrave PA. The amino-terminal tryptic peptide of bovine rhodopsin: a glycopeptide containing two sites of oligosaccharide attachment. Biochim Biophys Acta. 1977;492(1):83-94.

17. Fishman GA, Stone EM, Sheffield VC, Gilbert LD, Kimura AE. Ocular findings associated with rhodopsin gene codon 17 and codon 182 transition mutations in dominant retinitis pigmentosa. Arch Ophthalmol. 1992;110(1):54-62.

18. Sullivan LJ, Makris GS, Dickinson P, et al. A new codon 15 rhodopsin gene mutation in autosomal dominant retinitis pigmentosa is associated with sectorial disease. Arch Ophthalmol. 1993;111(11): 1512-1517.

19. Tam BM, Moritz OL. The role of rhodopsin glycosylation in protein folding, trafficking, and light-sensitive retinal degeneration. J Neurosci. 2009;29(48):15145-15154.

20. Fliesler SJ, Basinger SF. Tunicamycin blocks the incorporation of opsin into retinal rod outer segment membranes. Proc Natl Acad Sci U S A. 1985;82(4):1116-1120

21. Tam BM, Moritz OL. Dark rearing rescues $\mathrm{P} 23 \mathrm{H}$ rhodopsin-induced retinal degeneration in a transgenic Xenopus laevis model of retinitis pigmentosa: a chromophore-dependent mechanism characterized by production of $\mathrm{N}$-terminally truncated mutant rhodopsin. J Neurosci. 2007;27(34):9043-9053.

22. Tsybovsky Y, Molday RS, Palczewski K. The ATP-binding cassette transporter ABCA4 structural and functional properties and role in retinal disease. Adv Exp Med Biol. 2010;703:105-125.

23. Xu M, Yamada T, Sun Z, et al. Mutations in POMGNT1 cause non-syndromic retinitis pigmentosa. Hum Mol Genet. 2016;25(8):1479-1488.

24. Lam BL, Züchner SL, Dallman J, et al. Mutation K42E in dehydrodolichol diphosphate synthase (DHDDS) causes recessive retinitis pigmentosa. Adv Exp Med Biol. 2014;801:165-170.

25. Najmabadi H, Hu H, Garshasbi M, et al. Deep sequencing reveals 50 novel genes for recessive cognitive disorders. Nature. 2011;478(7367):57-63.

26. Timal S, Hoischen A, Lehle L, et al. Gene identification in the congenital disorders of glycosylation type I by whole-exome sequencing. Hum Mol Genet. 2012;21(19):4151-4161. 